\title{
Associação entre aspectos físicos-funcionais, comportamentais e de saúde com o medo de cair em idosos comunitários
}

\section{Association between physical-functional aspects, behavioral and health with fear of falling in community-dwelling older adults}

\author{
Jaquelini Betta Canever ${ }^{1}$, Ana Lúcia Danielewicz ${ }^{1}$, (D) Núbia Carelli Pereira de Avelar ${ }^{1}$
}

Departamento de Ciências da Saúde, Universidade Federal de Santa Catarina - UFSC

Correspondência

Núbia Carelli Pereira de Avelar

E-mail: nubia.carelli@ufsc.br

Submetido: 20 Outubro 2020

Aceito: 03 Novembro 2020

Como citar

Canever JB, Danielewicz AL, Avelar NCP. Associação entre aspectos físicos-funcionais, comportamentais e de saúde com o medo de cair em idosos comunitários. Acta Fisiatr. 2020;27(3):160-166

DOI: 10.11606/issn.2317-0190.v27i3a176447

\section{RESUMO}

O medo em cair é definido como uma preocupação exacerbada em cair na realização das atividades cotidianas. O conhecimento dos fatores associados ao medo de cair pode contribuir na elaboração de estratégias multidimensionais que visem reduzir futuras quedas nos idosos. Objetivo: Verificar a associação dos aspectos físicos-funcionais, comportamentais e de saúde com o medo de cair em idosos comunitários. Método: Tratou-se de um estudo transversal, com amostra aleatória probabilística, incluindo 308 idosos comunitários de ambos os sexos. A variável desfecho foi o medo em cair e as variáveis preditoras foram os aspectos comportamentais avaliados pelo autorrelato (histórico de quedas e autopercepção em saúde) e aspecto em saúde (sintomas depressivos). Já os aspectos físicos-funcionais incluíram a sarcopenia provável e a mobilidade. Resultados: Maiores chances de apresentar medo em cair foram observados em idosos que tiveram histórico de quedas (OR: 1,87; IC95\%: 1,10; 3,19), autopercepção negativa de saúde (OR: 3,35; IC95\%: 1,87; 6,00), sintomas depressivos (OR: 3,24; IC95\%: 1,88; 5,57), sarcopenia (OR: 4,22; IC95\%: 2,51; 7,09) e baixa mobilidade (OR: 4,19; IC95\%: $2,19 ; 7,99)$ quando comparados aos que não tinham as mesmas condições. Conclusão: Histórico de quedas, autopercepção ruim de saúde, sintomas depressivos, sarcopenia e baixa mobilidade foram associados ao medo de cair em idosos comunitários.

Palavras-chave: Acidentes por Quedas, Fatores de Risco, Envelhecimento

\begin{abstract}
Fear of falling is defined as an exacerbated concern in carrying out daily activities. Knowledge of factors associated with fear of falling may contribute to the development of multidimensional strategies that aim to reduce future falls in the elderly. Objective: To verify the association of physical-functional, health and behavioral aspects with the fear of falling of community-dwelling older adults. Methods: This was a cross-sectional study with a probabilistic random sample composed of 308 community-dwelling older men and women. The outcome variable was fear of falling and the predictor variables were behavioral aspects assessed by self-report (history of falls and self-perceived health) and health aspect (depressive symptoms). The physical-functional aspects included probable sarcopenia and mobility. Results: Higher chances of being afraid of falling were observed in the elderly who had a history of falls (OR: $1.87 ; 95 \% \mathrm{Cl}: 1.10 ; 3.19$ ), negative selfperceived health (OR: $3.35 ; 95 \% \mathrm{Cl}: 1.87 ; 6.00$ ), depressive symptoms (OR: $3.24 ; 95 \% \mathrm{Cl}$ : 1.88; 5.57), sarcopenia (OR: $4.22 ; 95 \% \mathrm{Cl}: 2.51 ; 7.09$ ) and low mobility (OR: $4.19 ; 95 \% \mathrm{Cl}$ : $2.19 ; 7.99)$ when compared to those who did not have the same conditions. Conclusion: History of falls, poor self-perception of health, depressive symptoms, sarcopenia and low mobility were associated with fear of falling in elderly community members.
\end{abstract}

Keywords: Accidental Falls, Risk Factors, Aging 


\section{INTRODUÇÃO}

As quedas corporais em idosos são um problema em saúde pública, pois repercutem em lesões, hospitalizações, mortalidade prematura e perda da independência funcional. ${ }^{1-3}$ Além disso, idosos com histórico de quedas, tendem a ter mais medo em cair, ${ }^{4}$ que é definido como uma preocupação exacerbada na realização das atividades de vida diária e que acarreta restrições na amplitude de movimento, diminuição na qualidade muscular, ${ }^{5}$ declínio no equilíbrio corporal, aumento no risco de futuras quedas, ${ }^{6}$ predisposição a ansiedade e depressão, $^{7}$ e consequente aumento na incapacidade funcional. ${ }^{3}$

Todas essas repercussões do medo de cair aumentam os gastos em saúde pública, considerando-se o aumento na utilização de fármacos ansiolíticos, ${ }^{8}$ internações e possíveis intervenções médicas. ${ }^{2} \mathrm{O}$ medo em cair tem alta prevalência em idosos. ${ }^{4}$ Scheffer et al. ${ }^{9}$ encontraram prevalência variando entre $21 \%$ a $85 \%$ em idosos comunitários na Europa e em território nacional, os valores variam entre $90,4 \%{ }^{10}$ e $95,2 \% .{ }^{11}$

Destaca-se que esses dados podem ser subestimados na prática clínica, uma vez que essas questões não são abordadas rotineiramente na avaliação dos idosos, resultando em oportunidades perdidas de prevenção e intervenção precoce.

Como o medo em cair contribui para o desenvolvimento de limitações funcionais, alguns estudos procuram definir condições associadas. ${ }^{2,9,12}$ Scheffer et al. ${ }^{9}$ e Rivasi et al. ${ }^{13}$ verificaram a associação de sintomas depressivos ao medo em cair. Por sua vez, Vitorino et al. ${ }^{2}$ associaram o medo em cair ao gênero feminino e também a autopercepção negativa de saúde. Outros estudos ainda avaliaram a associação entre o medo de cair e aspectos físicos funcionais, como a mobilidade, em idosos comunitários. ${ }^{14}$

Assim, o conhecimento dos fatores associados ao medo em cair pode contribuir para elaboração de estratégias multidimensionais que visem reduzir futuras quedas nos idosos comunitários. $^{9} \quad$ Apesar das abordagens anteriores semelhantes. ${ }^{2,15,16}$

\section{OBJETIVO}

Verificar a associação dos aspectos físicos-funcionais, de saúde e comportamentais com o medo de cair em idosos comunitários.

\section{MÉTODOS}

Tratou-se de um estudo transversal, com amostra aleatória probabilística, de base domiciliar, realizado com idosos (60 anos ou mais) de ambos os sexos, cadastrados no sistema de informação em saúde da Atenção Básica municipal do Balneário Arroio do Silva/SC - Sistema de Gestão Estratégica da Saúde (SIGES) (Produzido pela Branet, Tubarão, SC, Brasil). Este estudo está de acordo com princípios éticos contidos na Declaração de Helsinki.

O município Balneário Arroio do Silva está localizado na região sul do estado de Santa Catarina, pertencente a mesorregião sul. A população total residente de acordo com os dados do último Censo Demográfico era de 9.586 habitantes, dos quais $15,79 \%$ eram idosos. ${ }^{17}$ De acordo com o Programa das Nações Unidas para o Desenvolvimento ${ }^{18}$ o Índice de
Desenvolvimento Humano Municipal (IDHM) é 0,746, o que situa esse município na faixa de desenvolvimento humano alto. A dimensão que mais contribuiu para o IDHM do município foi a longevidade, com índice de 0,858 .

O cálculo amostral levou em consideração o total da população idosa cadastrada nas três Unidades Básicas de Saúde do município ( $n=2833$ ). Para o cálculo amostral foi considerada a prevalência para os desfechos desconhecida de $50 \%$, nível de confiança de $95 \%$ e erro amostral de seis pontos percentuais, estimando-se assim a necessidade de 302 voluntários para o estudo. Prevendo-se eventuais perdas amostrais foram elegíveis para compor a amostra 540 idosos.

Foram excluídos os idosos acamados, dependentes ou que não puderam responder aos questionários, residentes em instituições de longa permanência ou que haviam mudado o endereço residencial. Foram consideradas perdas os idosos não localizados em seus domicílios após três tentativas realizadas em dias e horários distintos e recusas aqueles que não aceitaram participar do estudo.

Os idosos participantes receberam orientações sobre os objetivos da pesquisa e assinaram o Termo de Consentimento Livre e Esclarecido. Este estudo recebeu aprovação pelo Comitê de Ética em Pesquisa com Seres Humanos da Universidade Federal de Santa Catarina (CAAE n-87776318.3.0000.0121).

A coleta dos dados foi realizada entre os meses de setembro de 2018 a setembro de 2019. Inicialmente, os idosos selecionados foram contatados via telefone e convidados a participarem do estudo, sendo então agendadas as visitas aos seus domicílios. Todos os entrevistadores foram previamente treinados, para padronização dos métodos de avaliação das medidas objetivas a serem realizados.

Os seguintes dados para caracterização da amostra foram coletados: sexo (Feminino e masculino), escolaridade (anos de estudo), renda mensal (salário), peso corporal (kilos), estatura (cm), Índice de Massa corporal (IMC) (kgm2), Circunferência da Cintura (cm), Estado Civil (Casado, Solteiro, Viúvo, Divorciado, Com Companheiro), Percepção em Saúde (Muito boa, Boa, Regular, Ruim, Muito ruim), Histório de Queda no ano Anterior (Sim e Não).

O medo em cair foi avaliado pelo Falls Efficacy Scale I (FESI), desenvolvido pela Rede Europeia de prevenção de quedas, ${ }^{19}$ traduzido e adaptado para idosos comunitários brasileiros. ${ }^{20}$ Este instrumento avalia a preocupação em sofrer queda ao realizar 16 atividades funcionais. As pontuações variam de 1 a 4 , sendo que quanto menor a pontuação, maior será o medo de cair. Os idosos com pontuação igual ou maior que 23 pontos foram categorizados com medo de cair. ${ }^{20}$

Histórico de quedas: Os idosos foram questionados sobre o histórico de quedas sofridas nos últimos 12 meses e foram categorizados com ou sem histórico de quedas. ${ }^{21}$

A ocorrência da queda foi definida como o evento caracterizado pelo deslocamento não intencional do corpo, da posição ortostática para um nível inferior, determinado por circunstâncias multifatoriais.

Avaliação da autopercepção de saúde: Foi questionado ao indivíduo como o mesmo percebia sua condição de saúde, tendo como alternativas de respostas cinco opções: 1) muito boa, 2) boa, 3) regular, 4) ruim e 5) muito ruim. Para as análises, os idosos foram agrupados em três categorias (muito boa/boa; regular; ruim/muito ruim). ${ }^{22,23}$ 
Sintomas depressivos: Os sintomas depressivos foram avaliados pela Escala de Depressão Geriátrica Abreviada (GDS15) desenvolvida por Yesavage \& Sheikh. ${ }^{24}$

No Brasil, sua adaptação transcultural e validação foi feita por Pereira ${ }^{26}$ e apresentou $92,70 \%$ de sensibilidade, $65,20 \%$ especificidade e 0,81 confiabilidade. A versão reduzida do questionário contém 15 questões na qual para cada resposta que corresponder a um sintoma depressivo deve-se atribuir 1 ponto. A pontuação total é o somatório de toda as respostas com características de sintomas depressivos. A categorização de sintomas depressivos foi realizada utilizando os pontos de corte maiores ou iguais a 6 pontos. ${ }^{27}$

Sarcopenia provável: A avaliação diagnóstica da sarcopenia provável foi realizada conforme atualizações descritas pelo European Working Group on Sarcopenia in Older People (EWGSOP2) com o uso do teste de sentar e levantar da cadeira de 5 repetições (TSLC5rep). O TSLC5rep foi realizado mensurando-se o tempo que o idoso levou para sentar e levantar da cadeira por 5 repetições com os braços cruzados sobre o peito. Aqueles que fizeram as cinco repetições em um tempo > 15 segundos foram classificados como sarcopênicos prováveis.

Mobilidade: A mobilidade foi avaliada pelo Timed Get Up and Go (TGUG), que quantifica o tempo despendido em um percurso de 6 metros: levantar de uma cadeira, deambular, contornar um obstáculo, retornar o trajeto e sentar. ${ }^{28}$

A realização do teste inicia com o avaliado sentado em postura ereta, com as mãos cruzadas sobre o tórax e pés apoiados no solo. Ao comando "Vá" emitido pelo avaliador, indivíduo levanta-se da cadeira e caminha até um ponto demarcado, contornando o mesmo, retorna e senta na cadeira o mais rápido possível. ${ }^{29-31}$
A cronometragem do tempo inicia quando o avaliado retira seu corpo do encosto da cadeira e é finalizada quando a toca novamente. ${ }^{28}$ Os idosos foram classificados com baixo desempenho quando os valores de realização do teste foram $\geq$ 12,47 segundos. $^{32}$

As análises foram ajustadas para sexo (feminino e masculino), faixa etária (60-69 anos; 70-79 anos e mais de 80 anos) e presença de multimorbidade considerando-se duas ou mais condições clínicas autorrelatadas (doença de coluna ou costas, artrite ou reumatismo, câncer, diabetes, bronquite ou asma, doença do coração ou cardiovascular, insuficiência renal crônica, tuberculose, cirrose, derrame/acidente vascular cerebral/isquemia cerebral, osteoporose, hipertensão, labirintite e incontinência urinária ou fecal. ${ }^{17}$

Os dados foram tabulados de forma independente por dois pesquisadores no software Excel, e posteriormente, foram

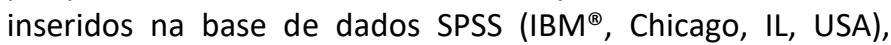
versão 23.0. O nível de significância adotado foi de 5\%.

Foram realizadas análises descritivas e apresentados os valores das proporções (\%) e respectivos intervalos de confiança de $95 \%$ (IC95\%). Para testar as associações entre as variáveis preditoras e o medo em cair foram realizadas análises de Regressão Logística Multivariada, estimando-se as razões de chances (OR) brutas e ajustadas, com seus respectivos intervalos de confiança (IC95\%).

\section{RESULTADOS}

Foram avaliados 308 idosos comunitários sendo 178 mulheres $(57.8 \%)$ e 130 homens $(42,2 \%)$ com média de idade de $69,67 \pm 6,99$ anos. Os resultados referentes a caracterização da amostra estão apresentados na Tabela 1.

Tabela 1. Características sociodemográficas, antropométricas e de saúde em idosos comunitários

\begin{tabular}{|c|c|c|c|c|}
\hline \multicolumn{2}{|c|}{ Variáveis } & \multirow{2}{*}{$\begin{array}{c}\text { Amostra geral } \\
\text { N (\%) Média } \pm \text { desvio- } \\
\text { padrão }\end{array}$} & \multirow{2}{*}{$\begin{array}{c}\begin{array}{c}\text { Com medo em cair } \\
\text { N (\%) Média } \pm \text { desvio- } \\
\text { padrão }\end{array} \\
105(59,0 \%)\end{array}$} & \multirow{2}{*}{$\begin{array}{c}\begin{array}{c}\text { Sem medo em cair } \\
\text { N (\%) Média } \pm \text { desvio- } \\
\text { padrão }\end{array} \\
73(41,0 \%)\end{array}$} \\
\hline Sexo & Feminino & & & \\
\hline & Masculino & $130(42,2 \%)$ & $44(33,8 \%)$ & $86(66,2 \%)$ \\
\hline & Idade (anos) & $69,67 \pm 6,99$ & $68,89 \pm 7,21$ & $70,04 \pm 6,87$ \\
\hline & Escolaridade (anos/estudo) & $5,68 \pm 3,76$ & $5,71 \pm 3,73$ & $5,67 \pm 3,78$ \\
\hline & Renda mensal (salário) & $1,94 \pm 1,58$ & $1,64 \pm 1,27$ & $2,09 \pm 1,70$ \\
\hline & Peso (kilos) & $74,95 \pm 16,27$ & $75,88 \pm 14,99$ & $74,51 \pm 16,86$ \\
\hline & Estatura (cm) & $160,56 \pm 9,02$ & $159,47 \pm 8,56$ & $161,08 \pm 9,21$ \\
\hline & IMC (kgm2) & $29,06 \pm 5,83$ & $29,96 \pm 6,20$ & $28,63 \pm 5,64$ \\
\hline & Circunferência da cintura & $100,88 \pm 12,81$ & $102,58 \pm 11,94$ & $100,10 \pm 13,16$ \\
\hline & Solteiro & $13(4,2 \%)$ & $8(61,5 \%)$ & $5(38,5 \%)$ \\
\hline \multirow{5}{*}{ Estado Civil } & Viúvo & $71(23,1 \%)$ & $41(57,7 \%)$ & $30(42,3 \%)$ \\
\hline & Divorciado & $42(13,6 \%)$ & $17(40,5 \%)$ & $25(59,5 \%)$ \\
\hline & Com companheiro & $8(2,6 \%)$ & $3(37,5 \%)$ & $5(62,5 \%)$ \\
\hline & Muito boa & $19(6,2 \%)$ & $2(10,5 \%)$ & $17(89,5 \%)$ \\
\hline & Boa & $97(31,5 \%)$ & $27(27,8 \%)$ & $70(72,2 \%)$ \\
\hline \multirow[t]{3}{*}{ Percepção em Saúde } & Regular & $135(43,8 \%)$ & $79(58,5 \%)$ & $56(41,5 \%)$ \\
\hline & Ruim & $35(11,4 \%)$ & $26(74,3 \%)$ & $9(25,7)$ \\
\hline & Muito ruim & $14(4,5 \%)$ & $10(71,4 \%)$ & $4(28,6 \%)$ \\
\hline \multirow{4}{*}{ Queda no ano Anterior } & Sim & $206(66,9 \%)$ & $83(40,3 \%)$ & $123(59,7 \%)$ \\
\hline & Não & $100(32,5 \%)$ & $64(64,0 \%)$ & $36(36,0 \%)$ \\
\hline & 60-64 anos & $82(26,6 \%)$ & $39(47,6 \%)$ & $43(52,4 \%)$ \\
\hline & 65-69 anos & $86(27,9 \%)$ & $36(41,9 \%)$ & $50(58,1 \%)$ \\
\hline \multirow[t]{3}{*}{ Idade } & 70-74 anos & $57(18,5 \%)$ & $27(47,4 \%)$ & $30(52,6 \%)$ \\
\hline & 75-79 anos & $52(16,9 \%)$ & $25(48,1 \%)$ & $27(51,9 \%)$ \\
\hline & 80 anos ou mais & $31(10,1 \%)$ & $22(71,0 \%)$ & $9(29,0 \%)$ \\
\hline \multirow{2}{*}{ Morbidades } & Com morbidades & $246(79,9 \%)$ & $138(56,1 \%)$ & $108(43,9 \%)$ \\
\hline & Sem morbidades & $62(20,1 \%)$ & $11(17,7 \%)$ & $51(82,3 \%)$ \\
\hline
\end{tabular}


Na análise de regressão logística, no modelo ajustado, observaram-se chances significativamente maiores de apresentar medo em cair entre os idosos que tiveram histórico de quedas (OR: 1,87; IC95\%: 1,10; 3,19), autopercepção ruim de saúde (OR: 3,35 ; IC95\%: 1,87; 6,00), sintomas depressivos (OR: 3,24; IC95\%: 1,88; 5,57), sarcopenia provável (OR: 4,22; IC95\%: 2,$51 ; 7,09$ ) e baixa mobilidade (OR: 4,19; IC95\%: 2,19; $7,99)$ quando comparados aos que não tinham as mesmas condições (Tabela 2).

Tabela 2. Análise de Regressão Logística Multivariável entre as variáveis preditoras e o medo em cair nos idosos amostrados

\begin{tabular}{|c|c|c|}
\hline \multirow{3}{*}{ Variáveis Preditoras } & \multicolumn{2}{|c|}{ Medo em cair } \\
\hline & Bruta & Ajustada \\
\hline & OR (IC 95\%) & OR (IC 95\%) \\
\hline \multicolumn{3}{|l|}{ Variáveis comportamentais } \\
\hline \multicolumn{3}{|l|}{ Histórico de quedas } \\
\hline Não & 1 & 1 \\
\hline Sim & $2,63(1,60 ; 4,31)$ & $1,87(1,10 ; 3,19)$ \\
\hline \multicolumn{3}{|l|}{ Percepção Saúde } \\
\hline Muito boa/boa ou regular & 1 & 1 \\
\hline Ruim / Muito ruim & $4,23(2,46 ; 7,27)$ & $3,35(1,87 ; 6,00)$ \\
\hline \multicolumn{3}{|l|}{ Variáveis físico-funcionais } \\
\hline \multicolumn{3}{|l|}{ Sarcopenia Provável } \\
\hline Não & 1 & 1 \\
\hline Sim & $4,94(3,04 ; 8,02)$ & $4,22(2,51 ; 7,09)$ \\
\hline \multicolumn{3}{|l|}{ Mobilidade } \\
\hline Alto desempenho & 1 & 1 \\
\hline Baixo desempenho & $5,32(2,91 ; 9,71)$ & $4,19(2,19 ; 7,99)$ \\
\hline \multicolumn{3}{|l|}{ Variáveis de Saúde } \\
\hline \multicolumn{3}{|l|}{ Sintomas depressivos } \\
\hline Não & 1 & 1 \\
\hline Sim & $3,61(2,18 ; 5,97)$ & $3,24(1,88 ; 5,57)$ \\
\hline
\end{tabular}

\section{DISCUSSÃO}

Os resultados do presente estudo demonstraram que os idosos com histórico de quedas, sarcopenia provável, sintomas depressivos, percepção em saúde ruim e baixa mobilidade tiveram chances significativamente maiores de apresentar medo em cair quando comparados aos que não relataram as mesmas condições. Referente ao histórico de quedas e sua associação com o medo em cair, diversos estudos relatam essa associação. ${ }^{4,33-36}$

Lavedán et al. ${ }^{4}$ mostraram que idosos com histórico prévio de quedas obtiveram 2,5 vezes mais chances de declararem medo em cair do que idosos sem tal condição.

No estudo de Kim \& So ${ }^{37}$ os autores observaram que idosos comunitários que já caíram pelo menos uma vez apresentam 6,41 vezes mais chances de relatar medo em cair. Os autores inferem que experiências negativas relacionadas à queda, tais como lesões e possíveis internações, afetam a percepção de autoeficiência, o que pode levar a efeitos negativos na função emocional e locomotora. ${ }^{36,38}$

Outro fator avaliado neste estudo foi a associação entre a sarcopenia provável e o medo em cair. Dados similares foram observados por alguns estudos ${ }^{39-41}$ e confirmaram o risco aumentado do medo em cair em idosos com sarcopenia. Uma possível hipótese relacionada a essa associação deve-se ao menor desempenho físico e as alterações resultantes da sarcopenia na capacidade física, já que se trata de uma condição que leva a perda da força e massa muscular e acarreta problemas ao equilíbrio corporal estático e dinâmico..$^{42}$

Os sintomas depressivos também esteve associado ao medo em cair em diversos estudos. ${ }^{3,39,41,43,44}$ Merchant et al. ${ }^{39}$ e Kirk ${ }^{41}$ demonstraram que idosos com sintomas depressivos apresentaram 4,90 (IC 1,06;22,67) e 4,11 (IC 2,31;7,29) maiores probabilidades em cair do que idosos sem sintomas depressivos, respectivamente.

Os autores justificam que a possível restrição física e insegurança para as quedas diminuem as atividades funcionais e predispõem a sintomas de depressão e ansiedade. Os idosos com depressão ${ }^{45-47}$ costumam ter prejuízos no desempenho físico, sobretudo dos músculos dos membros inferiores, além de maior tendência a comportamentos sedentários, o quais podem aumentar o medo em cair. ${ }^{48}$

A autopercepção negativa em saúde também foi associada com o medo em cair no presente estudo. Dados concordantes foram observados nos estudos. ${ }^{2,15,22,23,38,49}$ No estudo de Vitorino $^{2}$ realizado com idosos comunitários brasileiros, foi destacado que aqueles com baixa percepção em saúde apresentaram mais medo em cair do que aqueles com melhor percepção em saúde. Ademais, parece haver relação entre baixa percepção em saúde e maior número de doenças, ${ }^{50}$ fragilidade ${ }^{51}$ e diagnóstico de doenças mentais, ${ }^{52}$ o que pode influenciar no medo em cair.

$A$ associação entre o medo em cair e a mobilidade vem sendo abordada em diversos estudos. ${ }^{29,32,35,38,42,53-55}$ Alguns estudos destacaram também que a mobilidade está relacionada às limitações nas atividades funcionais de vida diária $^{56}$ e baixa mobilidade. ${ }^{55} \mathrm{O}$ baixo desempenho no TGUG em idosos com medo em cair pode ser explicado devido a sequência de movimentos do teste, o qual requer coordenação entre os membros e mudanças rápidas na orientação o que será realizado com maior cautela em um paciente com medo em cair e consequentemente demandando maior tempo para realização. ${ }^{57}$

Dentre as limitações deste estudo destacamos que amostra se limitou a um município do sul catarinense podendo não ser representativa de todo o território nacional.

Os achados deste estudo podem contribuir para a elaboração de ações modificar os fatores de risco e assim diminuir o medo em cair em idosos comunitários. Para estudos futuros, sugere-se a realização de estudos longitudinais em amostras maiores, para identificar outros fatores de risco associados ao medo em cair.

\section{CONCLUSÃO}

Histórico de quedas no último ano, autopercepção ruim de saúde, sintomas depressivos, sarcopenia provável e baixa mobilidade foram associados ao medo em cair em idosos comunitários.

\section{REFERENCIAS}

1. Gazibara T, Kurtagic I, Kisic-Tepavcevic D, Nurkovic S, Kovacevic N, Gazibara T, et al. Falls, risk factors and fear of falling among persons older than 65 years of age. Psychogeriatrics. 2017;17(4):215-23. Doi: https://doi.org/10.1111/psyg.12217 
2. Vitorino LM, Teixeira CA, Boas EL, Pereira RL, Santos NO, Rozendo CA. Fear of falling in older adults living at home: associated factors. Rev Esc Enferm USP. 2017;51:e03215. Doi: $\underline{220 \times 2016011803215}$

3. Chen SK, Voaklander D, Perry D, Jones CA. Falls and fear of falling in older adults with total joint arthroplasty: a scoping review. BMC Musculoskelet Disord. 2019;20(1):599. Doi: https://doi.org/10.1186/s12891019-2954-9

4. Lavedán $A$, Viladrosa $M$, Jürschik $P$, Botigué $T$, Nuín $C$, Masot $\mathrm{O}$, Lavedán $\mathrm{R}$. Fear of falling in community-dwelling older adults: A cause of falls, a consequence, or both? PLoS One. 2018;13(3):e0194967. Doi: https://doi.org/10.1371/journal.pone.0194967

5. Lee J, Choi M, Kim CO. Falls, a fear of falling and related factors in older adults with complex chronic disease. J Clin Nurs. 2017;26(23-24):4964-4972.

Doi: https://doi.org/10.1111/jocn.13995

6. Kovács É, Erdős Réka L, Petridisz Anna N, Rozs F, Simon A. Fear of falling among community-living older adults. Orvosi Hetilap. 2019;160(5):191-7. Doi: https://doi.org/10.1556/650.2019.31267.

7. Kendrick D, Kumar A, Carpenter H, Zijlstra GAR, Skelton D, Cook JR, et al. Exercise for reducing fear of falling in older people living in the community: Cochrane systematic review and Meta-Analysis. Age Ageing. 2016;45(3):34552. Doi: https://doi.org/10.1093/ageing/afw036

8. Lord SR, Close JCT. New horizons in falls prevention. Age Ageing. 2018;47(4):492-8. Doi: https://doi.org/10.1093/ageing/afy059.

9. Scheffer AC, Schuurmans MJ, Van dijk N, Van der hooft T, De rooij SE. Fear of falling: Measurement strategy, prevalence, risk factors and consequences among older persons. Age Ageing. 2008;37(1):19-24. Doi: https://doi.org/10.1093/ageing/afm169

10. Lopes KT, Costa DF, Santos LF, Castro DP, Bastone AC. Prevalência do medo de cair em uma população de idosos da comunidade e sua correlação com mobilidade, equilíbrio dinâmico, risco e histórico de quedas. Re Bras Fisioter. 2009;13(3):223-9. Doi: https://doi.org/10.1590/S1413-35552009005000026

11. Cruz DT, Duque RO, Leite ICG. Prevalência do medo de cair em uma população de idosos da comunidade. Rev Bras Geriatr Gerontol. 2017;20(3):309-18. Doi: https://doi.org/10.1590/1981-22562017020.160176

12. Heslop KR, Wynaden DG. Impact of falls on mental health outcomes for older adult mental health patients: An Australian study. Int J Ment Health Nurs. 2016;25(1):3-11. Doi: https://doi.org/10.1111/inm.12164

13. Rivasi G, Kenny RA, Ungar A, Romero-Ortuno R. Predictors of incident fear of falling in community-dwelling older adults. J Am Med Dir Assoc. 2020;21(5):615-620. Doi: https://doi.org/10.1016/i.jamda.2019.08.020

14. Litwin H, Erlich B, Dunsky A. The complex association between fear of falling and mobility limitation in relation to late life falls: a SHARE-based analysis. J Aging Health. 2018;30(6):987-1008.

Doi:
15. Vitorino LM, Marques-Vieira C, Low G, Sousa L, Cruz JP. Fear of falling among Brazilian and Portuguese older adults. Int J Older People Nurs. 2019;14(2):e12230. Doi: https://doi.org/10.1111/opn.12230

16. Souza AQ, Pegorari MS, Nascimento JS, Oliveira PB, Tavares DMDS. Incidence and predictive factors of falls in community-dwelling elderly: a longitudinal study. Cien Saude Colet. 2019;24(9):3507-16. Doi: https://doi.org/10.1590/1413-81232018249.30512017

17. Instituto Brasileiro de Geografia e Estatística (IBGE). Pesquisa Nacional de Saúde 2013. Rio de Janeiro: IBGE; c2013 [cited 2020 Oct 14]. Available from: http://www.ibge.gov.br/home/estatistica/populacao/pn s/2013/

18. Programa das Nações Unidas para o Desenvolvimento (PNUD). Brasília (DF): PNUD; c2013 [cited 2020 Oct 14]. Avaible from: https://www.br.undp.org/

19. Yardley L, Beyer N, Hauer K, Kempen G, Piot-Ziegler C, Todd C. Development and initial validation of the Falls Efficacy Scale-International (FES-I). Age Ageing. 2005;34(6):614-9.

Doi:

https://doi.org/10.1093/ageing/afi196

20. Camargos FF, Dias RC, Dias JM, Freire MT. Cross-cultural adaptation and evaluation of the psychometric properties of the Falls Efficacy Scale-International Among Elderly Brazilians (FES-I-BRAZIL). Rev Bras Fisioter. 2010;14(3):237-43. Doi: https://doi.org/10.1590/S1413$\underline{35552010000300010}$

21. Liu-Ambrose T, Davis JC, Best JR, Dian L, Madden K, Cook $W$, et al. Effect of a home-based exercise program on subsequent falls among community-dwelling high-risk older adults after a fall: a randomized clinical trial. JAMA. 2019;321(21):2092-2100.

https://doi.org/10.1001/jama.2019.5795

22. Zanesco C, Bordin D, Santos CB, Müller EV, Fadel CB. Fatores que determinam a percepção negativa da saúde de idosos brasileiros. Rev Bras Geriatr Gerontol. 2018;21(3):283-92. Doi: https://doi.org/10.1590/1981$\underline{22562018021.170210}$

23. Pereira LV, Vasconcelos PP, Souza LA, Pereira GA, Nakatani AY, Bachion MM. Prevalence and intensity of chronic pain and self-perceived health among elderly people: a population-based study. Rev Lat Am Enfermagem. 2014;22(4):662-9. Doi: https://doi.org/10.1590/0104-1169.3591.2465

24. Yesavage JA, Sheikh JI. 9/Geriatric Depression Scale (GDS) recent evidence and development of a shorter version. Clinical Gerontologist. 1986;5(1-2):119-36. Doi: https://doi.org/10.1300/J018v05n01 09

25. Pereira KR. Adaptação transcultural e validação da escala de depressão geriátrica GDS-15 [Dissertação]. Uberaba: Universidade Federal do Triângulo Mineiro; 2017.

26. Almeida OP, Almeida SA. Short versions of the geriatric depression scale: a study of their validity for the diagnosis of a major depressive episode according to ICD-10 and DSM-IV. Int J Geriatr Psychiatry. 1999;14(10):858-65. Doi: https://doi.org/10.1002/(sici)10991166(199910)14:10<858::aid-gps35>3.0.co;2-8 
27. Moreno DH, Dias RS, Moreno RA. Transtorno do humor. Porto Alegre: Artmed; 2010.

28. Podsiadlo D, Richardson S. The timed "Up \& Go": a test of basic functional mobility for frail elderly persons. J Am Geriatr Soc. 1991;39(2):142-8. Doi: https://doi.org/10.1111/i.1532-5415.1991.tb01616.x

29. Hwang R, Morris NR, Mandrusiak A, Mudge A, Suna J, Adsett J, et al. Timed Up and Go Test: A reliable and valid test in patients with chronic heart failure. J Card Fail. 2016;22(8):646-50.

Doi: http://dx.doi.org/10.1016/j.cardfail.2015.09.018

30. Nunciato AC, Pereira BC, Borghi-Silva A. Métodos de Avaliação da Capacidade Física e Qualidade de Vida dos Idosos: Revisão de Literatura. Saúde Rev. 2012;12(32):418. Doi: https://doi.org/10.15600/22381244/sr.v12n32p41-48

31. Cabral ALL. Tradução e validação do teste Timed Up \& Go e sua correlação com diferentes alturas da cadeira [Dissertação]. Brasília: Universidade Católica de Brasília; 2011.

32. Alexandre TS, Meira DM, Rico NC, Mizuta SK. Accuracy of Timed Up and Go Test for screening risk of falls among community-dwelling elderly. Rev Bras Fisioter. 2012;16(5):381-8. Doi: https://doi.org/10.1590/s141335552012005000041

33. Hill KD. Fear of falling: A hidden burden with or without a history of falls. Evidence-Based Nursing. 2019;22:21. Doi: http://dx.doi.org/10.1136/eb-2018-102978

34. Oh E, Hong GS, Lee S, Han S. Fear of falling and its predictors among community-living older adults in Korea. Aging Ment Health. 2017;21(4):369-378. Doi: https://doi.org/10.1080/13607863.2015.1099034

35. Makino K, Makizako H, Doi T, Tsutsumimoto K, Hotta R, Nakakubo $S$, et al. Impact of fear of falling and fall history on disability incidence among older adults: Prospective cohort study. Int J Geriatr Psychiatry. 2018;33(4):658-62. Doi: https://doi.org/10.1002/gps.4837

36. Lee $\mathrm{S}, \mathrm{Oh} \mathrm{E}$, Hong GS. Comparison of factors associated with fear of falling between older adults with and without a fall history. Int J Environ Res Public Health. 2018;15(5):982.

https://doi.org/10.3390/ijerph15050982

37. Kim S, So WY. Prevalence and correlates of fear of falling in Korean community-dwelling elderly subjects. Exp Gerontol. 2013;48(11):1323-8. Doi: https://doi.org/10.1016/i.exger.2013.08.015

38. Hoang OT, Jullamate P, Piphatvanitcha N, Rosenberg E. Factors related to fear of falling among communitydwelling older adults. J Clin Nurs. 2017;26(1-2):68-76. Doi: https://doi.org/10.1111/jocn.13337

39. Merchant RA, Chen MZ, Wong BLL, Ng SE, Shirooka H, Lim $J Y$, et al. Relationship between fear of falling, fear-related activity restriction, frailty, and Sarcopenia. J Am Geriatr Soc. 2020. Doi: https://doi.org/10.1111/igs.16719

40. Sampaio RAC, Sampaio PYS, Castaño LAA, Barbieri JF, Coelho Júnior $\mathrm{HJ}$, Arai $\mathrm{H}$, et al. Cutoff values for appendicular skeletal muscle mass and strength in relation to fear of falling among Brazilian older adults: cross-sectional study. Sao Paulo Med J. 2017;135(5):43443. Doi:https://doi.org/10.1590/1516-3180.2017.0049030517
41. Kirk B, Zanker J, Bani Hassan E, Bird S, Brennan-Olsen S, Duque G. Sarcopenia definitions and outcomes consortium (sdoc) criteria are strongly associated with malnutrition, depression, falls, and fractures in high-risk older persons. J Am Med Dir Assoc. 2020:S15258610(20)30572-7.

https://doi.org/10.1016/i.jamda.2020.06.050

42. Gadelha AB, Neri SGR, Oliveira RJ, Bottaro M, David AC, Vainshelboim $B$, et al. Severity of sarcopenia is associated with postural balance and risk of falls in communitydwelling older women. Exp Aging Res. 2018;44(3):258269.

Doi:

https://doi.org/10.1080/0361073X.2018.1449591

43. Akın S, Mazıcıoglu MM, Mucuk S, Gocer S, Deniz Şafak E, Arguvanlı $S$, et al. The prevalence of frailty and related factors in community-dwelling Turkish elderly according to modified fried frailty index and FRAIL scales. Aging Clin Exp Res. 2015;27(5):703-9. Doi: https://doi.org/10.1007/s40520-015-0337-0

44. Ang GC, Low SL, How CH. Approach to falls among the elderly in the community. Singapore Med J. 2020;61(3):116-21.

Doi:

https://doi.org/10.11622/smedj.2020029

45. Brooks JM, Titus AJ, Bruce ML, Orzechowski NM, Mackenzie TA, Bartels SJ, et al. Depression and handgrip strength among U.S. adults aged 60 years and older from NHANES 2011-2014. J Nutr Health Aging. 2018;22(8):93843. Doi: https://doi.org/10.1007/s12603-018-1041-5

46. Lenze EJ, Schulz R, Martire LM, Zdaniuk B, Glass T, Kop WJ, et al. The course of functional decline in older people with persistently elevated depressive symptoms: longitudinal findings from the Cardiovascular Health Study. J Am Geriatr Soc. 2005;53(4):569-75. Doi: https://doi.org/10.1111/j.1532-5415.2005.53202.x

47. Esbrí-Víctor $M$, Huedo-Rodenas I, López-Utiel M, NavarroLópez JL, Martínez-Reig M, Serra-Rexach JA, et al. Frailty and Fear of Falling: The FISTAC Study. J Frailty Aging. 2017;6(3):136-40.

Doi: https://doi.org/10.14283/jfa.2017.19

48. Santos KT, Fernandes MH, Reis LA, Coqueiro RS, Rocha SV. Sintomas depressivos e desempenho motor em idosos: Estudo de base populacional. Rev Bras Fisioter. 2012;16(4):295-300. Doi: https://doi.org/10.1590/S1413$\underline{35552012005000030}$

49. Li Q, Mpofu E, Yin C, Turner KW. Perception of Falls and Confidence in Self-Management of Falls among Older Adults. Int J Environ Res Public Health. 2019;16(24):5054. Doi: https://doi.org/10.3390/ijerph16245054

50. Jerez-Roig J, Souza DL, Andrade FL, Lima Filho BF, Medeiros RJ, Oliveira NP, et al. Self-perceived health in institutionalized elderly. Cien Saude Colet. 2016;21(11):3367-75. Doi: https://doi.org/10.1590/1413812320152111.15562015

51. Medeiros SM, Silva LS, Carneiro JA, Ramos GC, Barbosa AT, Caldeira AP. Fatores associados à autopercepção negativa da saúde entre idosos não institucionalizados de Montes Claros, Brasil. Cien Saude Colet. 2016;21(11):3377-86. Doi: https://doi.org/10.1590/1413$\underline{812320152111.18752015}$ 
52. Fernandes MGM, Andrade AN, Nóbrega MML. Antecedents of frailty in the elderly: a systematic revision. OBJN 2010;9(1). Doi: https://doi.org/10.5935/16764285.20102847

53. Bjerk M, Brovold T, Skelton DA, Bergland A. Associations between health-related quality of life, physical function and fear of falling in older fallers receiving home care. BMC Geriatr. 2018;18(1):253. Doi: https://doi.org/10.1186/s12877-018-0945-6

54. Kyrdalen IL, Thingstad P, Sandvik L, Ormstad H. Associations between gait speed and well-known fall risk factors among community-dwelling older adults. Physiother Res Int. 2019;24(1):e1743. Doi: https://doi.org/10.1002/pri.1743

55. Williams JM, Nyman SR. Association between the instrumented timed up and go test and cognitive function, fear of falling and quality of life in community dwelling people with dementia. J Frailty Sarcopenia Falls. 2018;3(4):185-93. Doi: https://doi.org/10.22540/JFSF-03$\underline{185}$
56. Kwan MM, Lin SI, Chen CH, Close JC, Lord SR. Sensorimotor function, balance abilities and pain influence Timed Up and Go performance in older community-living people. Aging Clin Exp Res. 2011;23(3):196-201. https://doi.org/10.1007/BF03324960

57. Galán-Mercant A, Cuesta-Vargas Al. Clinical frailty syndrome assessment using inertial sensors embedded in smartphones. Physiol Meas. 2015;36(9):1929-42. Doi: https://doi.org/10.1088/0967-3334/36/9/1929 